\title{
Double-strand break repair processes drive evolution of the mitochondrial genome in Arabidopsis
}

Jaime I Davila', Maria P Arrieta-Montiel', Yashitola Wamboldt ${ }^{1}$, Jun Cao ${ }^{2}$, Joerg Hagmann², Vikas Shedge ${ }^{1,3}$, Ying-Zhi Xu' ${ }^{1}$, Detlef Weigel ${ }^{2}$ and Sally A Mackenzie ${ }^{1 *}$

\begin{abstract}
Background: The mitochondrial genome of higher plants is unusually dynamic, with recombination and nonhomologous end-joining (NHEJ) activities producing variability in size and organization. Plant mitochondrial DNA also generally displays much lower nucleotide substitution rates than mammalian or yeast systems. Arabidopsis displays these features and expedites characterization of the mitochondrial recombination surveillance gene MSH1 (MutS 1 homolog), lending itself to detailed study of de novo mitochondrial genome activity. In the present study, we investigated the underlying basis for unusual plant features as they contribute to rapid mitochondrial genome evolution.

Results: We obtained evidence of double-strand break (DSB) repair, including NHEJ, sequence deletions and mitochondrial asymmetric recombination activity in Arabidopsis wild-type and msh1 mutants on the basis of data generated by Illumina deep sequencing and confirmed by DNA gel blot analysis. On a larger scale, with mitochondrial comparisons across 72 Arabidopsis ecotypes, similar evidence of DSB repair activity differentiated ecotypes. Forty-seven repeat pairs were active in DNA exchange in the msh1 mutant. Recombination sites showed asymmetrical DNA exchange within lengths of 50- to 556-bp sharing sequence identity as low as $85 \%$. De novo asymmetrical recombination involved heteroduplex formation, gene conversion and mismatch repair activities. Substoichiometric shifting by asymmetrical exchange created the appearance of rapid sequence gain and loss in association with particular repeat classes.

Conclusions: Extensive mitochondrial genomic variation within a single plant species derives largely from DSB activity and its repair. Observed gene conversion and mismatch repair activity contribute to the low nucleotide substitution rates seen in these genomes. On a phenotypic level, these patterns of rearrangement likely contribute to the reproductive versatility of higher plants.
\end{abstract}

\section{Background}

While producing a circular genetic map, plant mitochondrial chromosomes comprise a heterogeneous population of highly branched, circularly permuted linear molecules $[1,2]$. In plants, large-sized mitochondrial repeats (more than 1,000 bp) conduct high-frequency reciprocal recombination to subdivide the genome [3]. Given the linear structure of the genome, this recombination activity is

\footnotetext{
* Correspondence: smackenzie2@unl.edu

'Center for Plant Science Innovation, University of Nebraska, N305 Beadle Center, Lincoln, NE 68588-0660, USA

Full list of author information is available at the end of the article
}

suspected to participate in recombination-mediated replication initiation $[4,5]$.

Mitochondrial genome plasticity is evident in extensive DNA polymorphism among closely related lines of a given plant species [6], rapid accumulation of polymorphisms within a line under cell culture conditions [7-9] and novel insertions, deletions and sequence chimeras [6]. These types of genomic alterations are causative in cytoplasmic male sterility (CMS) in a wide range of plant species [10], implying an adaptive rationale for the genomic phenomena [11]. Still, questions remain about the processes underlying mitochondrial genome variation, since most previous studies have addressed existing variations without

\section{Biomed Central}


the benefit of de novo events. Mitochondrial DNA replication in plants is not well-detailed, but both rolling circle and recombination-mediated replication have been suggested $[4,12,13]$. Likewise, the DNA repair mechanisms accounting for unusually low nucleotide substitution rates in plants are not known $[14,15]$.

Sufficient mitochondrial DNA has been extracted from plant tissues to suggest the presence of multiple copies of the genome per cell, but available data suggest that the genome is organized into a heterogeneous population of DNA molecules maintained at vastly different relative copy numbers. Some substoichiometric DNA configurations are stably maintained in the genome and transmitted to progeny at levels approximating 1 copy per 200 cells [16]. The term "substoichiometric shifting" (SSS) refers to the reversible interconversion of these low-level forms to high copy numbers, influencing gene expression in the process $[17,18]$. This intragenomic copy number shifting is rapid, likely occurs during plant reproduction [19] and involves differential recombination at intermediate-sized repeats within the genome [20,21].

Asymmetrical recombination is characterized by the accumulation of only one of the two predicted DNA exchange products. Intermediate repeats support asymmetrical recombination in the plant mitochondrial genome at extremely low levels. This recombination activity accounts for faint "ghost" band polymorphisms commonly reported in DNA gel blot hybridization experiments involving long film exposure times [20]. Disruption of the Arabidopsis nuclear gene MSH1 (MutS 1 homolog), a component of mitochondrial recombination surveillance [20,22], allows increased recombination activity at these repeats. Recent studies of mitochondrial genomic changes in the Arabidopsis msh1 mutant, performed using PCR and DNA gel blot analysis, permitted functional identification of 33 intermediate repeat pairs 108 to $556 \mathrm{bp}$ in size [21], implying a potential for massive rearrangement of the mitochondrial genome by this type of recombination activity. In "first-generation" $m s h 1$ mutants created by pollination of a wild-type line with mutant $m s h 1$ pollen and selection of the $\mathrm{F}_{2} m s h 1 / m s h 1$ segregant, low-level recombination activity is initiated at each repeat. By the "advanced generation," derived by recurrent self-pollination of the msh1 mutant, recombination is extensive and recombinants are present at high copy numbers [21].

Most previously acquired information about plant mitochondrial genome variation has relied on studies of CMS mutants and DNA polymorphism in natural populations. Discovery of $m s h 1$ permits direct investigation of mitochondrial de novo recombination and its relationship to high levels of structural variation and unusually low nucleotide substitution rates unique to plants. In our present study, next-generation sequencing provided sufficient sensitivity for detailed investigation of the asymmetrical strand invasion and gene conversion process as well as sufficient throughputfor cataloging genomic alterations associated with rapid, intraspecific mitochondrial genome evolution.

\section{Results}

Next-generation sequence analysis reveals additional intermediate repeats not previously detected

Mitochondrial asymmetric DNA exchange in the $m s h 1$ mutant involves 47 repeat pairs ranging from 50 to $556 \mathrm{bp}$ in size (Figure 1A). This was deduced by identification of paired adjacent sequences that were known to map distal to one another. Such paired-end sequences were clustered in $m s h 1$ first- and advanced-generation mutants in a manner distinct from the wild type (Additional file 1, Figure $\mathrm{S} 1$ ). All identified clusters could be explained by recombination events involving intermediate repeats. While confirming previously identified repeats [21], deep sequencing revealed 14 new repeat pairs ranging from 50 to $250 \mathrm{bp}$ in size, with sequence homology within each pair ranging from $85 \%$ to $98 \%$ and the smallest repeats displaying the highest identity (Figure 1B). All recombination detected at intermediate repeats showed asymmetrical exchange activity.

\section{Intermediate repeats reveal essential features of the mitochondrial DNA exchange process}

The size range of active repeats reflects the length of homology required to permit DNA exchange. While repeated sequences smaller than $50 \mathrm{bp}$ were detected in the genome, they showed no evidence of recombination activity, suggesting that $50 \mathrm{bp}$ approximates a lower size limit for $m s h 1$-associated DNA exchange. Recombination patterns at intermediate repeats imply a process of break-induced replication on the basis of the uniformly asymmetrical nature of DNA exchange [23,24]. Each repeat showed a conserved polarity for strand invasion, which was suggested by the accumulation of only one of the recombinant products and, in some cases, concomitant loss of one of the parental forms [21]. The observed polarity was confirmed by comparing high-throughput sequence frequencies at sites flanking repeats in firstand advanced-generation $m s h 1$ mutants with the wild type (Figure 2A; Additional file 2, Figure S2; and Additional file 3, Table S1). Analysis of clustered intermediate repeats allowed us to compare relative strand invasion polarities and showed both opposite and samestrand polarities at neighboring repeats, with no obvious association with local direction of transcription (Figure $2 \mathrm{~B})$. It is possible that these sites of active, bidirectional strand invasion might represent replication origins (for example, at sites between repeats $\mathrm{E}-2$ and $\mathrm{O}-2$ and between repeats $\mathrm{L}-1$ and $\mathrm{G}-2$ ). 


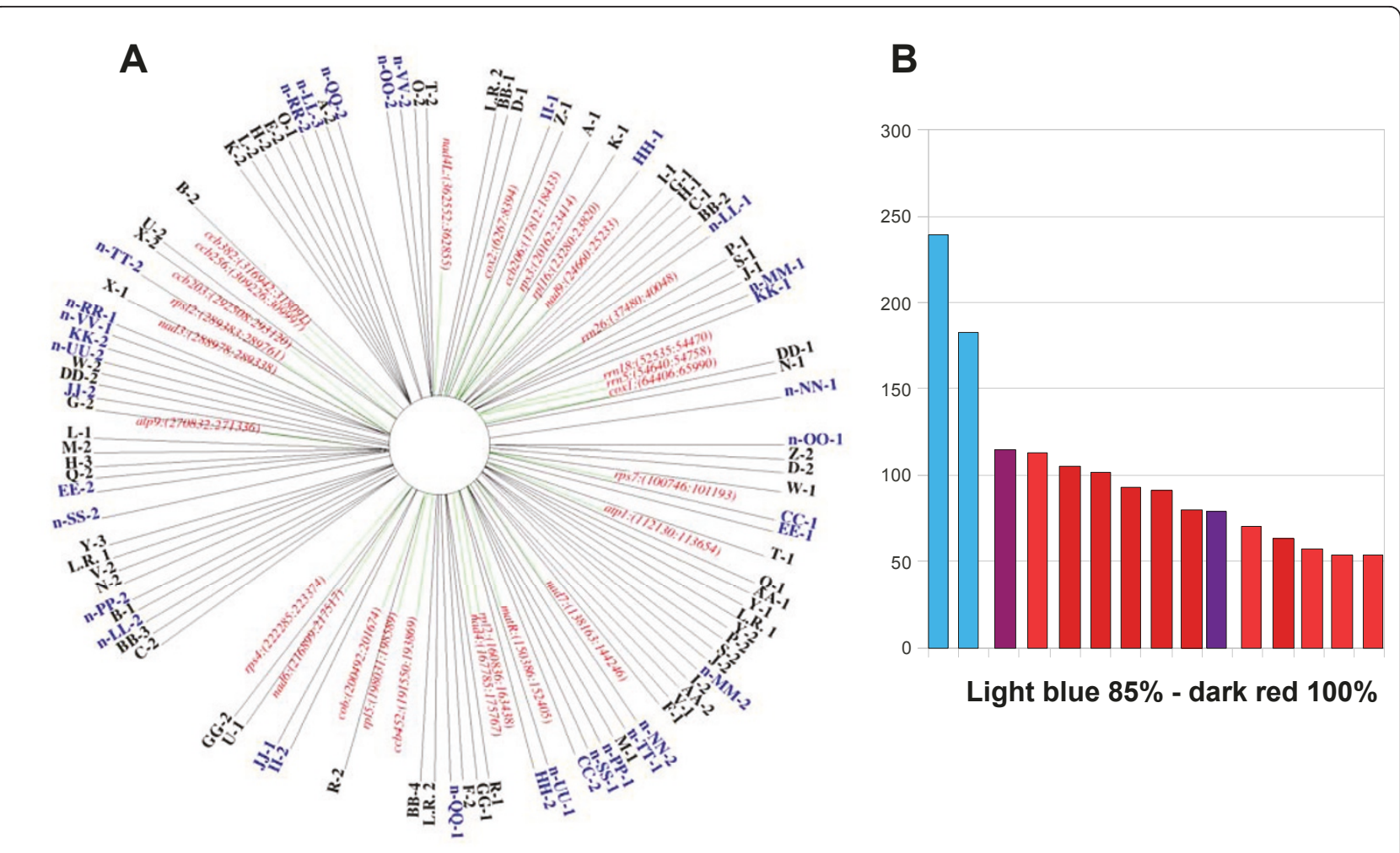

Figure 1 Identification of 14 additional Arabidopsis mitochondrial genome repeat pairs. (A) Map of intermediate mitochondrial repeats that are active in the msh1 mutant. Newly identified repeats are shown in blue. (B) Size (y-axis) and percentage homology (color) distribution of newly identified repeats.

Sequence analysis of the recombination product for nonidentical repeats, composing about $50 \%$ of all active intermediate repeats, provided evidence of heteroduplex formation followed by mismatch repair (Figures 3A and $3 \mathrm{~B})$. According to our data, nucleotide mismatches are repaired using the donor strand as a template. In Figure $3 \mathrm{~A}$, the nucleotide insertions in the donor strand are cleaved, giving the appearance of partial gene conversion. However, a more extensive survey of repeats showed no clear pattern of donor or recipient strand cleavage for correction of nucleotide insertions. These results reveal the presence of a mitochondrial mismatch repair mechanism in plants that is independent of MSH1.

To assess the influence of $m s h 1$ recombination on mitochondrial genome copy number, we compared relative copy number at 11 sites within the mitochondrial genome of the wild type (Col-0) and the msh1 mutant (Figure 4). In each case, copy number was higher in the mutant, implying that overall mitochondrial DNA copy number increases with enhanced recombination. One possible explanation for this observation might be enhanced break-induced replication of the genome in the absence of MSH1
Mitochondrial recombination in the msh 1 mutant produces two patterns of genomic rearrangement

We compared the read frequency for parental forms in different generations of the msh1 mutant against wild type. This allowed us to trace the fate of parental products over time in the msh1 mutant. Most repeats appeared to fall into one of two general categories (Figure 5). Class I repeats lost one parental form as they acquired the recombinant, resulting in the appearance of both deletion and gain of genomic forms. Class II repeats retained the parental forms as they acquired the recombinant, resulting in the appearance of a novel genomic form. The parental form that was reduced was consistently that which donates the invading strand.

This "gain" and "loss" of genetic environments is the consequence of SSS, and PCR-based analysis has revealed that these genomic environments change in relative copy number merely as a consequence of recombination $[20,21]$. From a genomic perspective, sequences residing in proximity to these intermediate repeats have the greatest likelihood of undergoing SSS, although we have identified sequences some distance from an identifiable repeat that also undergo SSS. We observed several essential genes residing in proximity to class II repeats (Table 1), but 


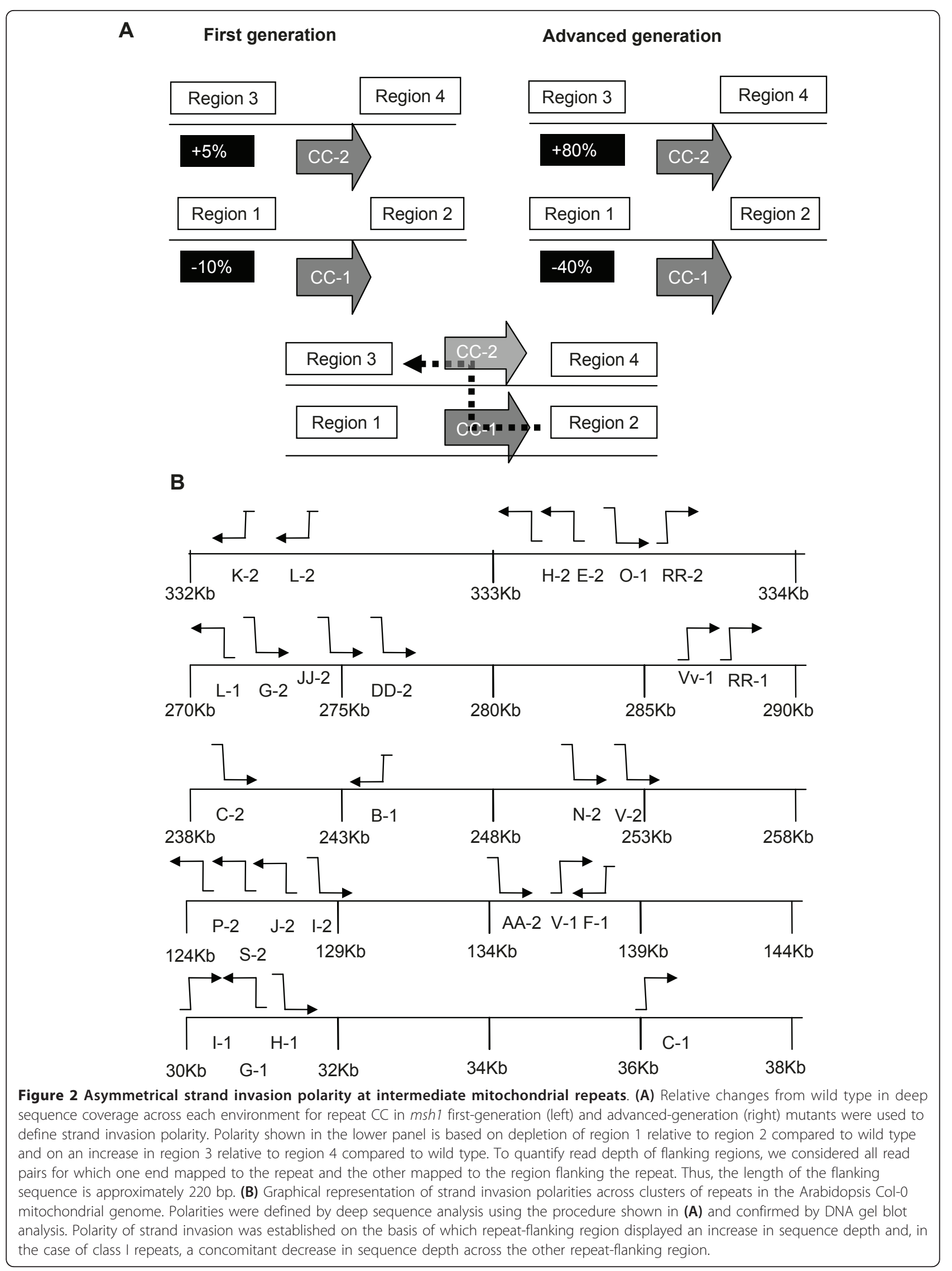




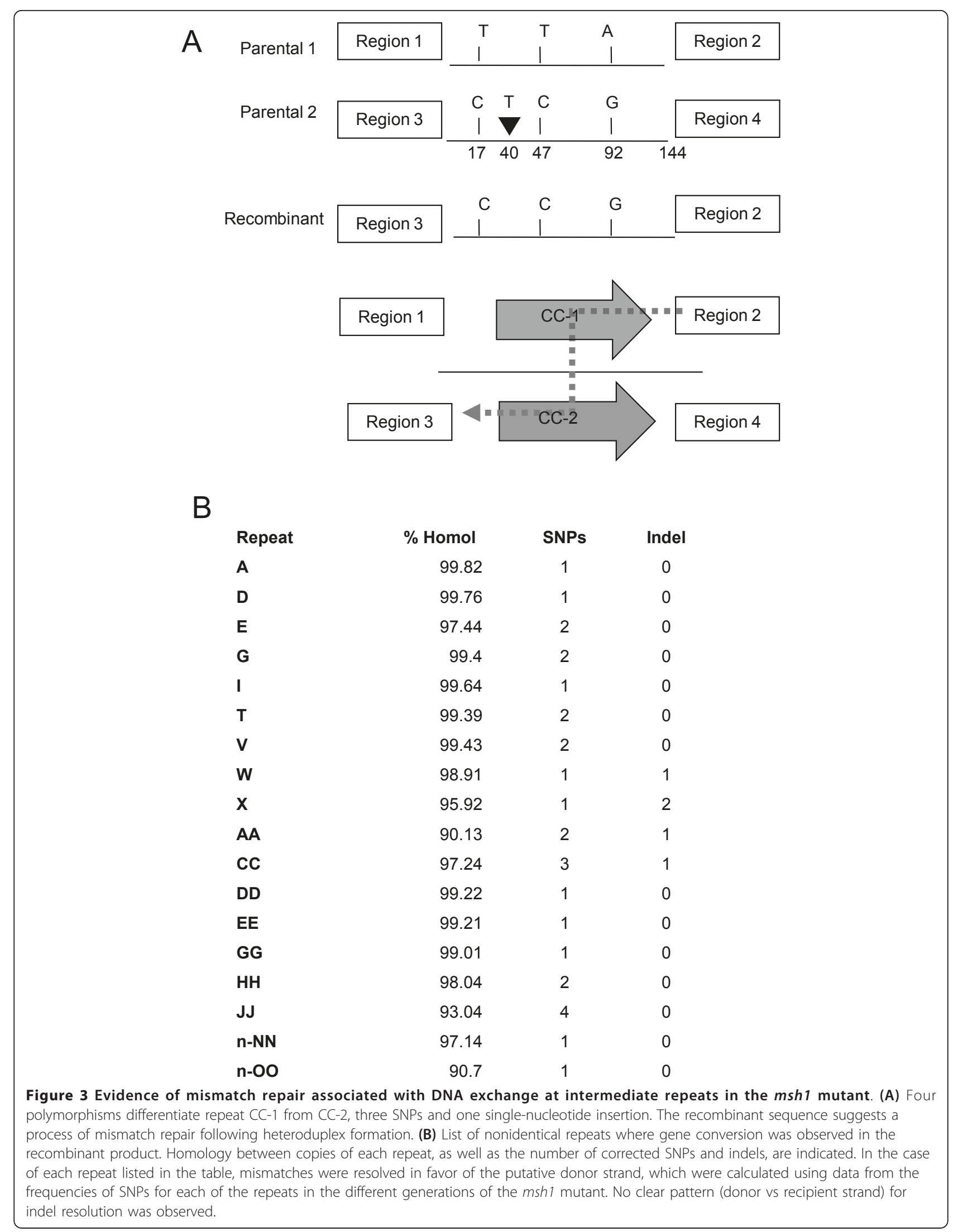




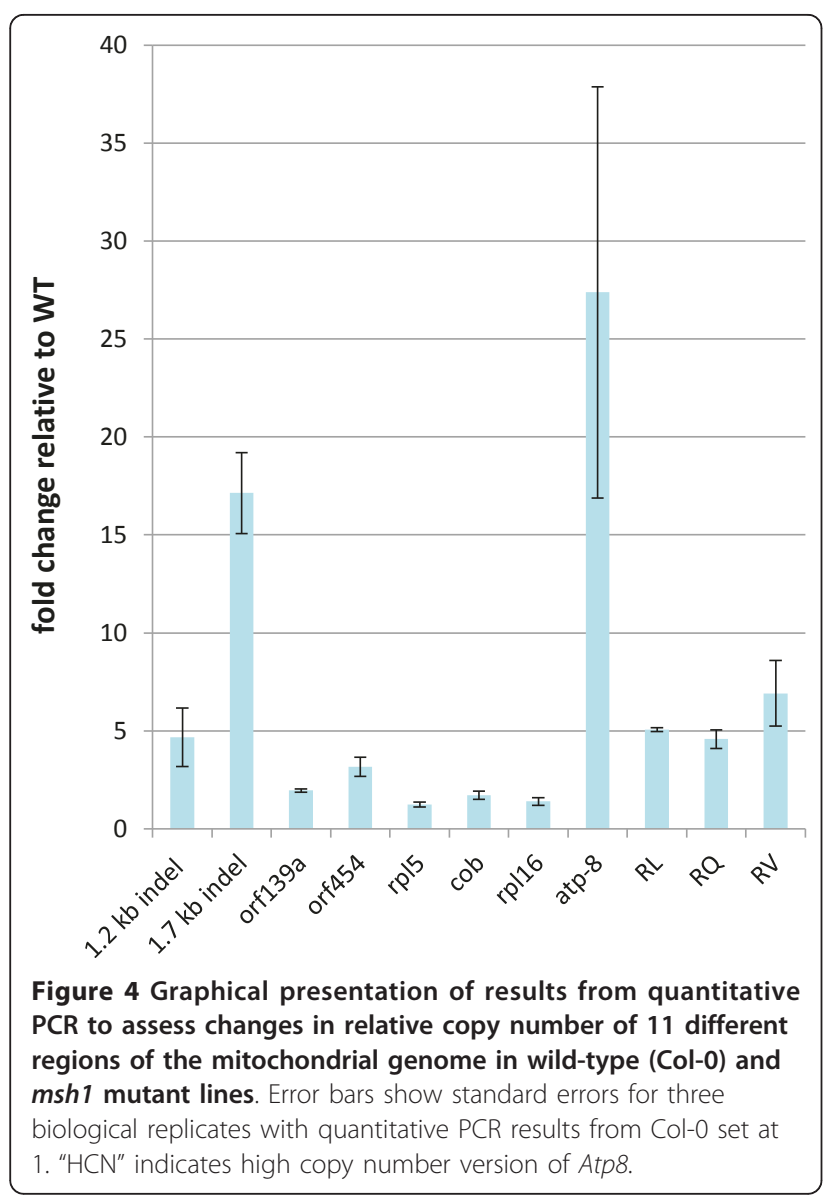

none residing adjacent to class I repeats (with the exception of Atp9, which encompasses a class I repeat). Presumably, the relative proximity of a repeat to essential genetic information could impose selection for the parental form, the class II pattern.

In msh1 advanced- versus first-generation mutants, we investigated the reversibility of recombination activity upon replacement of the MSH1allele. While first-generation $m s h 1$ rearrangements appeared to be fully reversible by crossing the $m s h 1$ line with the wild type and deriving an $F_{2}$ progeny, advanced-generation rearrangements were not reversible, based on analysis of 10 individual $F_{2}$ plants from both crosses and assaying three repeats ( $\mathrm{L}, \mathrm{D}$ and $\mathrm{F}$ ) for recombinant configurations (data not shown). We attribute these results to cytoplasmic sorting of the least prevalent forms once MSH1 is reintroduced. Thus, in the first generation, where the quantity of recombinant molecule is low relative to nonrecombinant, recombinant forms are diluted and nonrecombinant forms predominate. In advanced generations, where recombinant forms have accumulated to higher levels, their loss by sorting is precluded.
The Arabidopsis mitochondrial genome undergoes rapid and extensive rearrangement

In our investigation of the influence of double-strand break (DSB)-mediated changes on genomic variation, we sequenced and assembled the mitochondrial genomes of ecotypes Col-0 and Landsberg erecta (Ler) and compared these with the publicly available $\mathrm{C} 24$ sequence (confirmed by resequencing). Among the three ecotypes, we found virtually identical genomic coding capacity within genomes that had undergone extensive rearrangements. The majority of the structural polymorphisms found can be attributed to DSB repair activity by either nonhomologous end-joining (NHEJ) or intermediatesized repeat-mediated recombination (Figures $6 \mathrm{~A}$ and $6 \mathrm{~B})$.

Stoichiometric variation among ecotypes includes a repeat-containing interval of $1.7 \mathrm{~kb}$, originally reported by Forner et al. [25], that is present in Col-0 but substoichiometric in C24 and Ler [21]. The corresponding "junction" configuration that lacks this $1.7-\mathrm{kb}$ insertion is present in C24 and Ler, but is substoichiometric in Col-0. Likewise, an unrelated interval of $1.2 \mathrm{~kb}$ present in C24 and Ler was found to be substoichiometric in Col-0. The corresponding "junction" lacking this 1.2-kb interval is present in Col-0 but substoichiometric in C24 and Ler (data not shown).

While cross-ecotype genomic comparisons gave evidence of ecotype-specific divergence by insertion and deletion of distinct genetic information, this variation was largely accounted for by asymmetrical recombination-mediated SSS. Researchers in earlier cross-genotype studies in maize and sugar beet, involving multiple lines, reported similar mitochondrial variation $[6,26]$. While the researchers in neither of the earlier studies investigated substoichiometric forms in the genome, we suggest that some of the reported insertions and deletions distinguishing the closely related genotypes in those species likely derive from SSS activity.

The extensive mitochondrial DNA polymorphism among lines within a species was not surprising, given what has been reported in other plant studies [27]. What was significant in our investigation was the discovery that nearly all of the variation observed could be accounted for by DSB repair activity or nucleotide substitutions. This observation prompted us to investigate the extent to which DSB repair processes have participated in plant mitochondrial genome evolution.

\section{DSB repair occurs widely in Arabidopsis mitochondrial genomes}

To assess the role of DSB repair in mitochondrial genome evolution, we assembled and compared mitochondrial sequences from 72 different Arabidopsis ecotypes. 


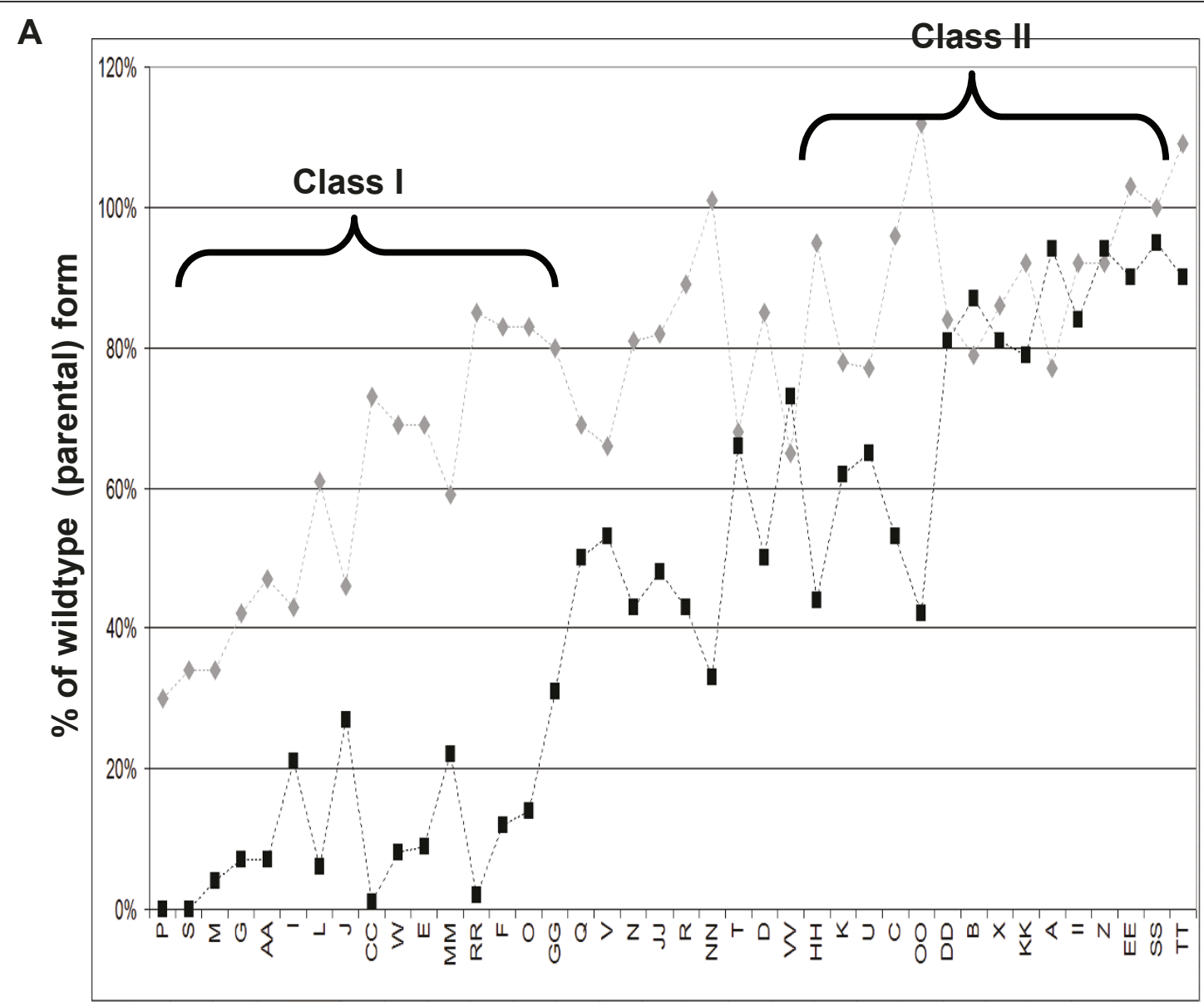

B

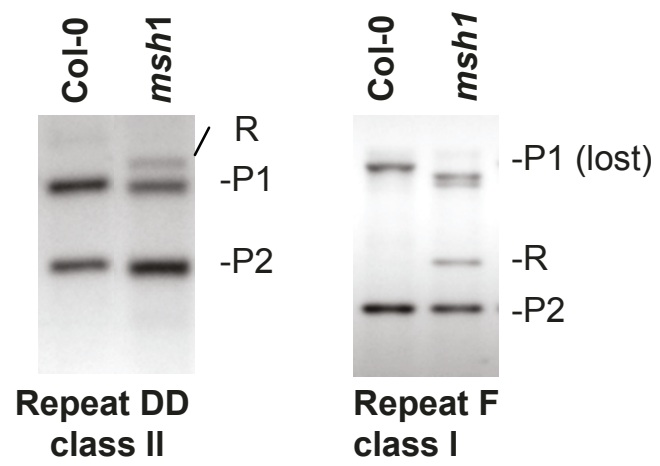

Figure 5 Deducing the fate of the parental form following recombination. (A) Graph depicting deep sequence coverage of the least predominant environment (the invading strand parental form) around each repeat. This is measured by quantifying the changes in read depth across flanking regions compared to wild type. Repeats were sorted by average activity, with first-generation msh1 repeats shown in gray and advanced-generation results shown in black. Class I repeats show significant reduction of the invading strand parental form in the resulting recombinant, and class II repeats retain the parental form in high copy numbers following recombination. Mapping of class I repeats gives the appearance of deletion in the recombinant. (B) DNA gel blot hybridization experiments with repeat DD and repeat F as probes show examples of class I and class II recombination outcomes in an advanced-generation msh1 mutant. P1 and P2 designate parental configurations, and R is the recombinant form. Additional bands in the $m s h 1$ lane probed with repeat F represent secondary recombinations, defined as events that depend on novel genomic environments created by primary recombination to occur. 
Table 1 Proximity of class II repeats to known genes

\begin{tabular}{llll}
\hline Repeat & Position & Gene & Gene position \\
\hline B & 322,365 & Cox3 & 330,907 \\
X & 289,252 & Nad3 & 288,978 \\
KK & 52,356 & Rrn26 & 40,047 \\
A & 19,679 & Rps3 & 23,280 \\
II & 210,316 & Nad6 & 216,899 \\
Z & 11,399 & Ccb206 & 17,812 \\
EE & 107,016 & Rps7 & 101,192 \\
SS & 263,498 & Atp6-1 & 253,200 \\
\hline
\end{tabular}

We collaborated with the Arabidopsis 1001 Genomes project (http://www.1001genomes.org/) to obtain mitochondrial sequences for subsequent identification of polymorphisms. The mitochondrial genome sequences were scanned for SNPs to assess phylogenetic relationships among the ecotypes. Cross-ecotype SNP comparisons suggested that the 72 ecotypes comprised six phylogenetic groups (Figure 7). The derived groupings appeared to correspond generally with the geographic origin of each line, and the variation measured is likely to reflect divergence over a span of 120,000 years [28]. This observation would be consistent with the observed sequence divergence across ecotypes, which was approximately one-twentieth that of Arabidopsis lyrata, with an estimated divergence time of four to five million years.

Mitochondrial structural polymorphisms revealed extensive genomic variation distinguishing each line. The majority of structural changes represented nonhomologous, recombination or small deletion activity (Figure 8). The small deletions were a small proportion (about 20\%) of the total structural polymorphisms detected. Crossecotype cataloging of observed structural variation revealed remarkable correspondence with the six SNPbased phylogenetic groups (Figure 8 ). These observations would be expected if DSB repair served as a prominent contributor to the rapid evolution rate of plant mitochondrial genomes.

\section{Discussion}

While we have not yet identified the genomic features that condition asymmetrical recombination in the Arabidopsis mitochondrial genome, we have shown in the present study that the process comprises the primary pattern of DNA exchange at intermediate-sized repeat sequences in the absence of $M S H 1$. It is possible that the observed asymmetrical exchange might be a consequence of differential maintenance of recombination products rather than the consequence of dominant strand invasion polarity. While it has been suggested that analysis of low-frequency recombination events can sometimes be confused with PCR-generated artefacts [29], our analysis of de novo recombination in $m s h 1$ confirms prolific DNA exchange activity. Data from our studies suggest that strand invasion occurs throughout the genome, a likely consequence of random DSBs, but is prevented by $M S H 1$ from producing successful DNA exchange in stretches of sequence homology under about $556 \mathrm{bp}$. In the absence of $M S H 1$, strand invasion proceeds at smaller lengths of homology to a lower limit of approximately $50 \mathrm{bp}$.

In the wild-type line, mitochondrial DNA exchange at intermediate repeats does occur, but at very low levels, presumably because it is prevented by MSH1 surveillance. This infrequent recombination is detected as faint ghost bands by DNA gel blot analysis [20,21]. MSH1 encodes a carboxy-terminal GIY-YIG endonuclease domain, although direct participation in double-strand cleavage to abort DNA exchange has not yet been formally tested. MSH1 presumably permits repair of a DSB at stretches of homology greater than $550 \mathrm{bp}$ following heteroduplex formation, assuming a break-induced replication model (see Additional file 4, Figure S3).

In the msh1 mutant, heteroduplex formation occurs within the intermediate repeat stretches of homology. The observation of heteroduplex-mediated mismatch repair in this study provides important evidence relevant to the low mitochondrial nucleotide substitution rates reported in plants [14]. Our study also shows that this mismatch repair activity is independent of $M S H 1$. Interestingly, nucleotide divergence within intermediate repeat intervals, estimated across 72 Arabidopsis ecotypes at 5.5 per 10,000 $\mathrm{bp}$, approximates the average for the genome as a whole, which is 5 per $10,000 \mathrm{bp}$. However, in regions of the genome where high-frequency reciprocal DNA exchange occurs, within large (more than 1,000 bp) repeats, levels of polymorphism were 66 times lower, averaging 7.5 per Mbp for the 6.5-kb repeat across 72 ecotypes. These observations imply that the low-frequency exchanges observed at intermediate repeats in the wild-type line may be sufficient to confer the low nucleotide substitution rate observed generally in the Arabidopsis mitochondrial genome. In animal lineages, nucleotide substitution rates in the mitochondrial genome are much higher, with the notable exception of corals and their relatives [30]. Intriguingly, the corals appear to be the only known animal lineage encoding a mitochondrial Msh1, with similarities in structure to MSH1 in plants [31].

Analysis of mitochondrial genomic rearrangements differentiating the Col-0, Ler and C24 ecotypes revealed three primary classes of genomic changes as recombination, NHEJ and deletion, all of which may represent consequences of DSB repair (Figure 9). An important aspect of NHEJ may be the formation of sequence chimeras, some of which encode transcriptionally active open reading frames that could condition phenotypic variations, such as CMS. Proximity of a CMS sequence chimera to a class I 


\section{A}
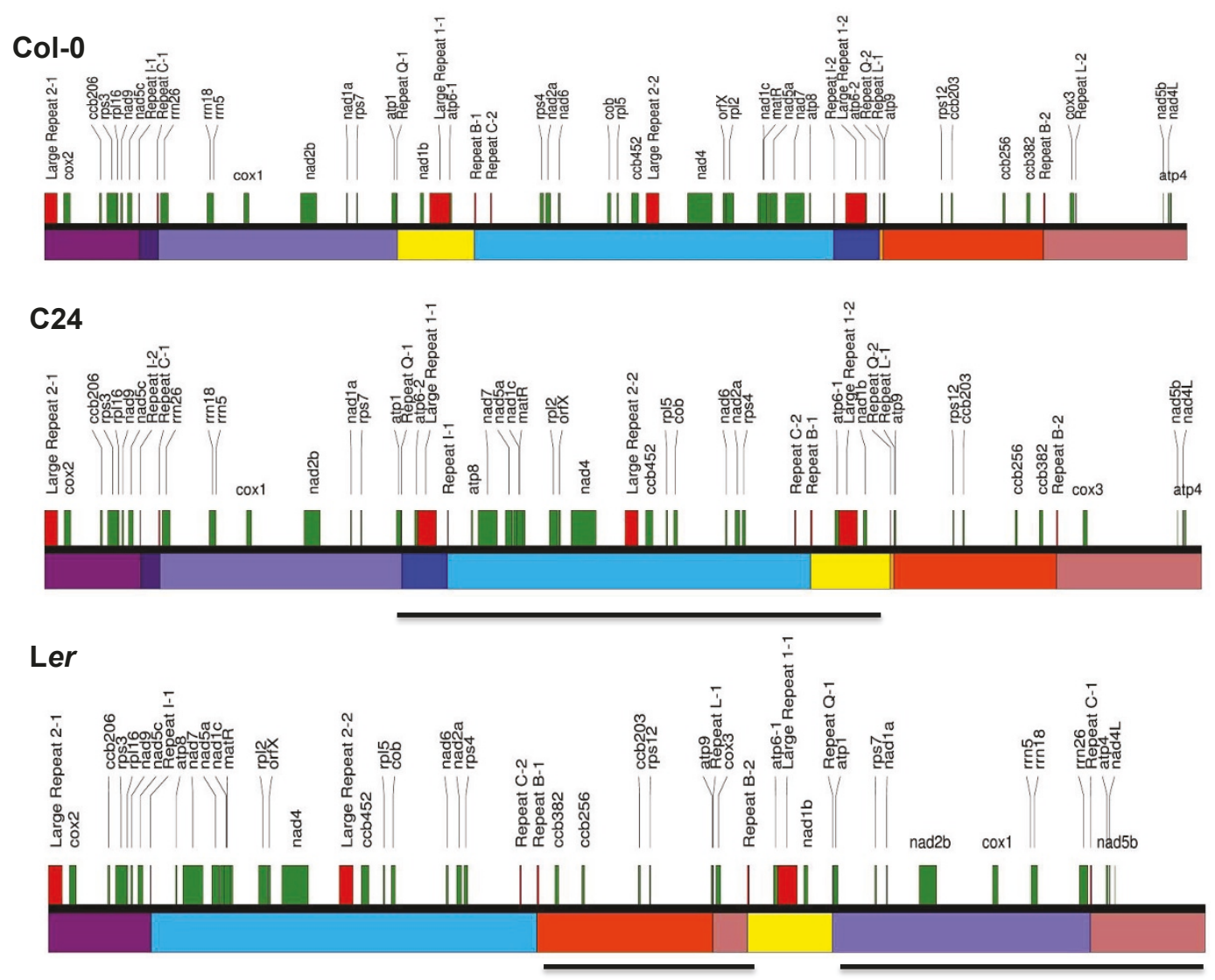

B

\begin{tabular}{c|c|c|c} 
Ecotype & Start & End & Type \\
\hline Col-0 & 217,704 & 98,986 & NHEJ \\
\hline Col-0 & 271,388 & 272,496 & Small deletion \\
\hline Col-0 & 280,353 & 301,934 & Reciprocal recombination around Q \\
\hline Ler & 12,087 & 98,693 & Asymmetric recombination around C \\
\hline Ler & 18,182 & 12,281 & Asymmetric recombination around I \\
\hline Ler & 280,353 & 301,394 & Asymmetric recombination around Q \\
\hline Ler & 279,353 & 107,067 & Asymmetric recombination around L \\
\hline Ler & 103,792 & 227,097 & Reciprocal recombination around B
\end{tabular}

Figure 6 Mitochondrial genome rearrangements in ecotypes Col-0, C24 and Ler. (A) Genome maps are constructed from sequence data. Regions are color-coded, with genes shown in green and selected repeats shown in red. Underlying bars represent regions that are inverted in orientation relative to the genomic map of Col-0. (B) List of structural variations in Col-0 and Landsberg erecta (Ler) genes relative to C24. The position and type of polymorphism are shown. 


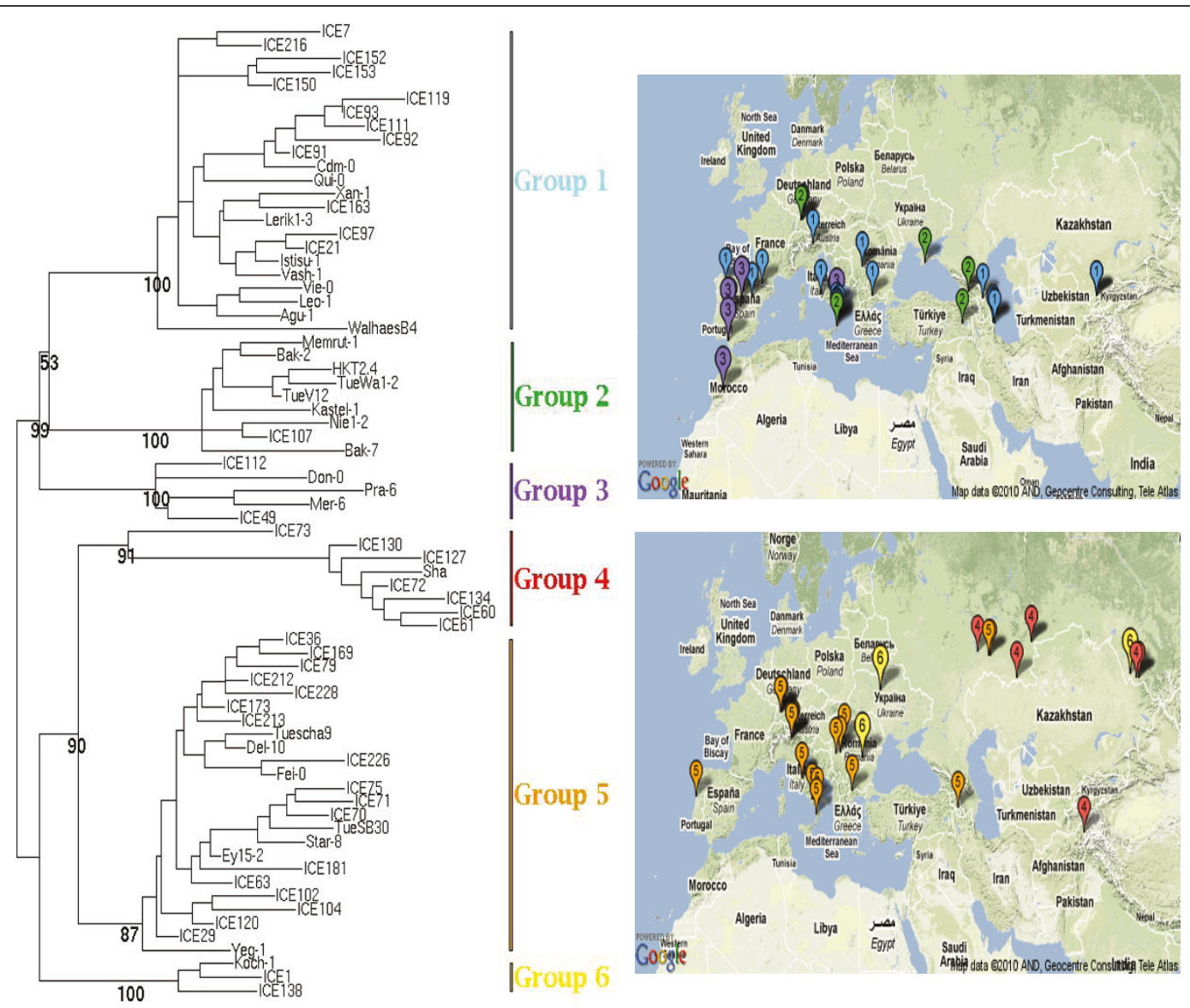

Figure 7 Phylogenetic analysis of 72 ecotypes based on mitochondrial SNPs suggests division into six groups in the left panel. The tree shown is unrooted. The right panel shows the geographic distribution of the depicted groups.

repeat can allow changes in copy number of the region by recombination at the repeat. CMS induction was shown to occur by MSH1 suppression in various plant species [32]. The resulting mitochondrial DNA rearrangements are presumed to result in copy number amplification of a novel, preexistent, CMS-inducing genomic environment.

SSS-associated copy number reduction of male sterility sequences has been associated with spontaneous reversion to fertility in common bean, Brassica, and maize Stype cytoplasm [18,33-35]. Whether the frequency of reversion responds to altered $M S H 1$ expression in the plant, and whether MSH1 expression is influenced by environmental cues such as unsuccessful pollination, is an intriguing possibility. MSH1 transcription is environmentally responsive [36], but it is not clear whether carbon flux acts as a signal. An isolated male-sterile plant, following a period of unsuccessful pollination, might affect carbon signaling to suppress $M S H 1$, amplifying mitochondrial recombination for SSS and reversion to fertility. Such a process, if it occurred late in the flowering cycle, would ensure a successful seed set.

\section{Conclusions}

In our present study, we conducted a detailed analysis of de novo recombination and genome rearrangement processes that characterize plant mitochondrial genomes. The rapid rate of plant mitochondrial intraspecific structural variation and low nucleotide substitution rates can both be accounted for by unusual DSB repair within the genome. These observations consequently implicate DSB repair processes as a driver in the evolutionary divergence of plant and animal mitochondria. The dynamic nature of plant mitochondrial genomes, and their ability to retain genetic variation substoichiometrically, presumably enhance environmental responsiveness and increase reproductive versatility.

\section{Methods}

\section{DNA gel blot and PCR assays}

Total genomic DNA was extracted from above-ground tissues according to previously published procedures [37]. Mitochondrial DNA preparation, DNA gel blot analysis and hybridizations were performed as described 


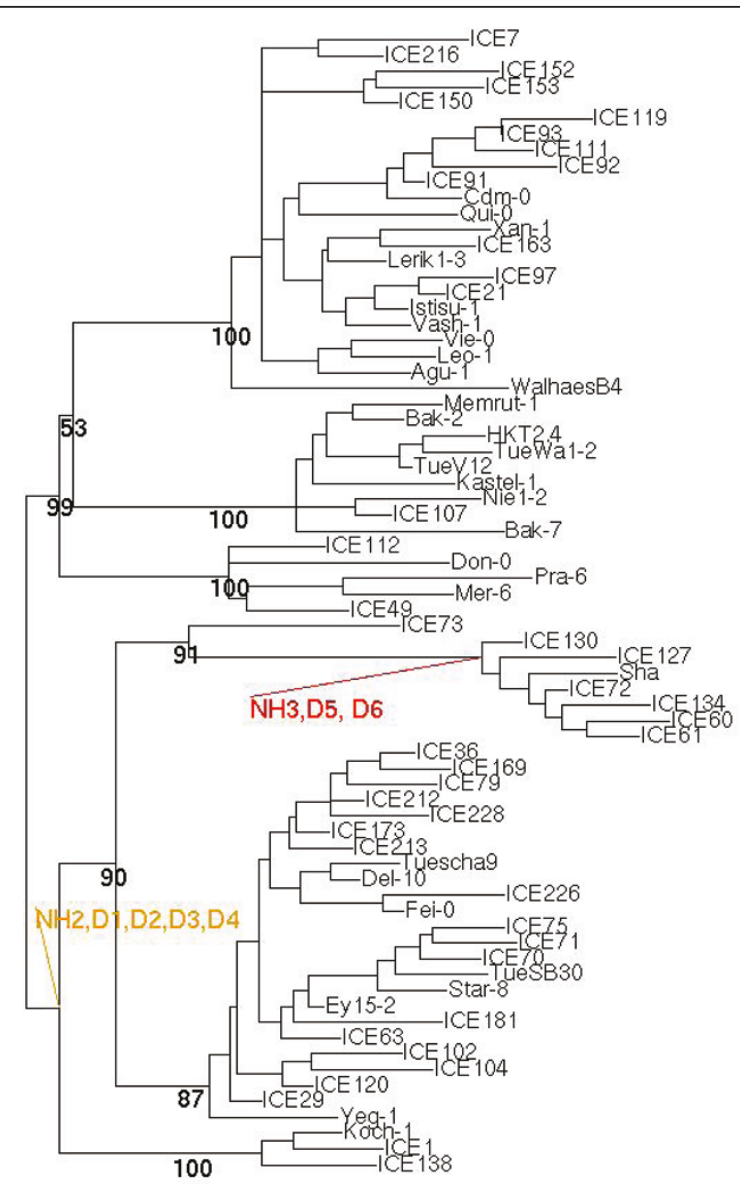

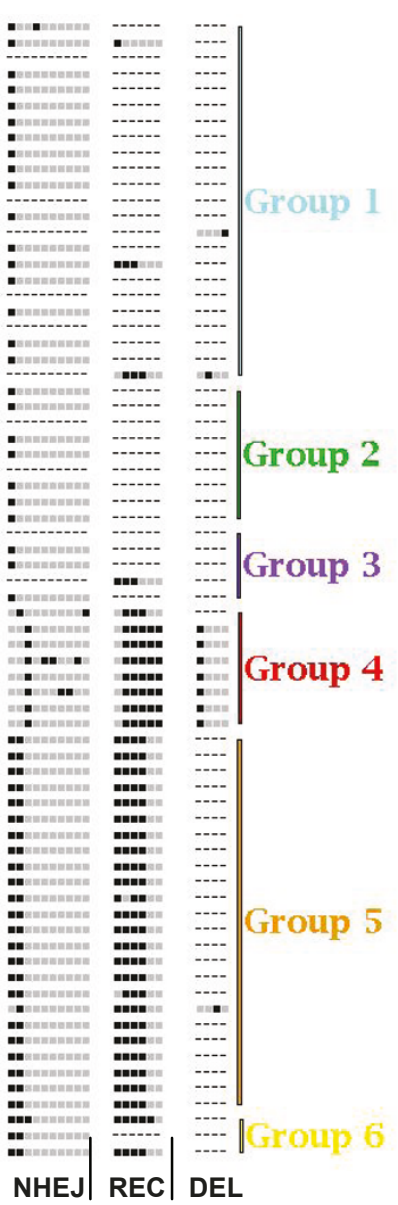

Figure 8 Mitochondrial genome structural changes coincide with SNP-based phylogeny. Structural changes arising by nonhomologous end-joining (NHEJ), deletions caused by recombination at repeats (REC) and small deletions, perhaps arising by replication slippage (DEL), were categorized according to ecotypes, allowing assessment of the relationship of SNP-based phylogeny to changes arising by genomic rearrangement. Putative sites in the tree where some of the changes may have occurred are indicated. The tree shown is unrooted.

previously [38]. The primers used to PCR-amplify probes are listed in Additional file 5, Table S2.

\section{Quantitative polymerase chain reaction}

Genomic DNA (100 ng) was used for quantitative PCR with the SYBR GreenER qPCR SuperMix for iCycler kit (Invitrogen, Carlsbad, CA, USA). Quantitative PCR data were collected and analyzed using iCycler iQ version 3.1 software (Bio-Rad Laboratories, Hercules, CA, USA). Three biological replicates were used for each experiment, each sample was run in triplicate and the results were averaged. Nuclear single-copy loci (AtMSH1 and At $A C$ TIN2) served as nuclear DNA content controls. The primers used for real-time analysis are presented in Additional file 5, Table S2.

\section{Sequencing and read mapping}

Illumina Solexa Sequencing Technology (Illumina, Inc, San Diego, CA, USA) paired-end reads of Col-0 were assembled using the comparative assembler AMOScmp version 2.08 software program (with default parameters) [39] with the C24 mitochondrial genomic sequence as a baseline to obtain 57 contigs with an N50 of $25 \mathrm{~kb}$ and coverage of $230 \times$. In a similar manner, C24 was resequenced and 54 contigs obtained, with an N50 of $30 \mathrm{~kb}$ and coverage of $371 \times$. Single and paired-end reads for Ler were mapped against the reference genome of C24 using Velvet version 1.1 software (with default parameters) [40] and SHOREmap version 0.5 software (with default parameters) [41] to obtain $19 \times$ coverage, and consensus sequences and deleted regions were identified using custom-made Perl scripts. The contigs for these three ecotypes were assembled into circular scaffolds based on DNA gel blot analysis and PCR assays. Graphical genome maps were generated using OGDraw software [42]. The annotated genome sequences for ecotypes C24, Ler and Col-0 are available from GenBank [GenBank:JF729200, GenBank:JF729201, GenBank:JF729202], 

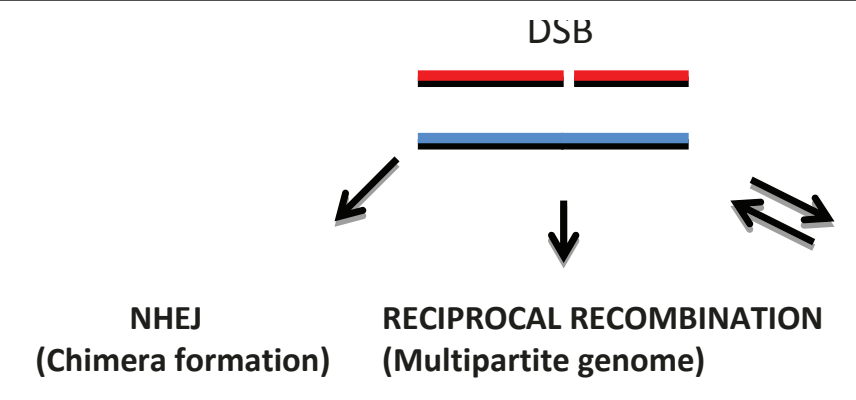

\section{ASYMMETRIC RECOMBINATION (AR)}
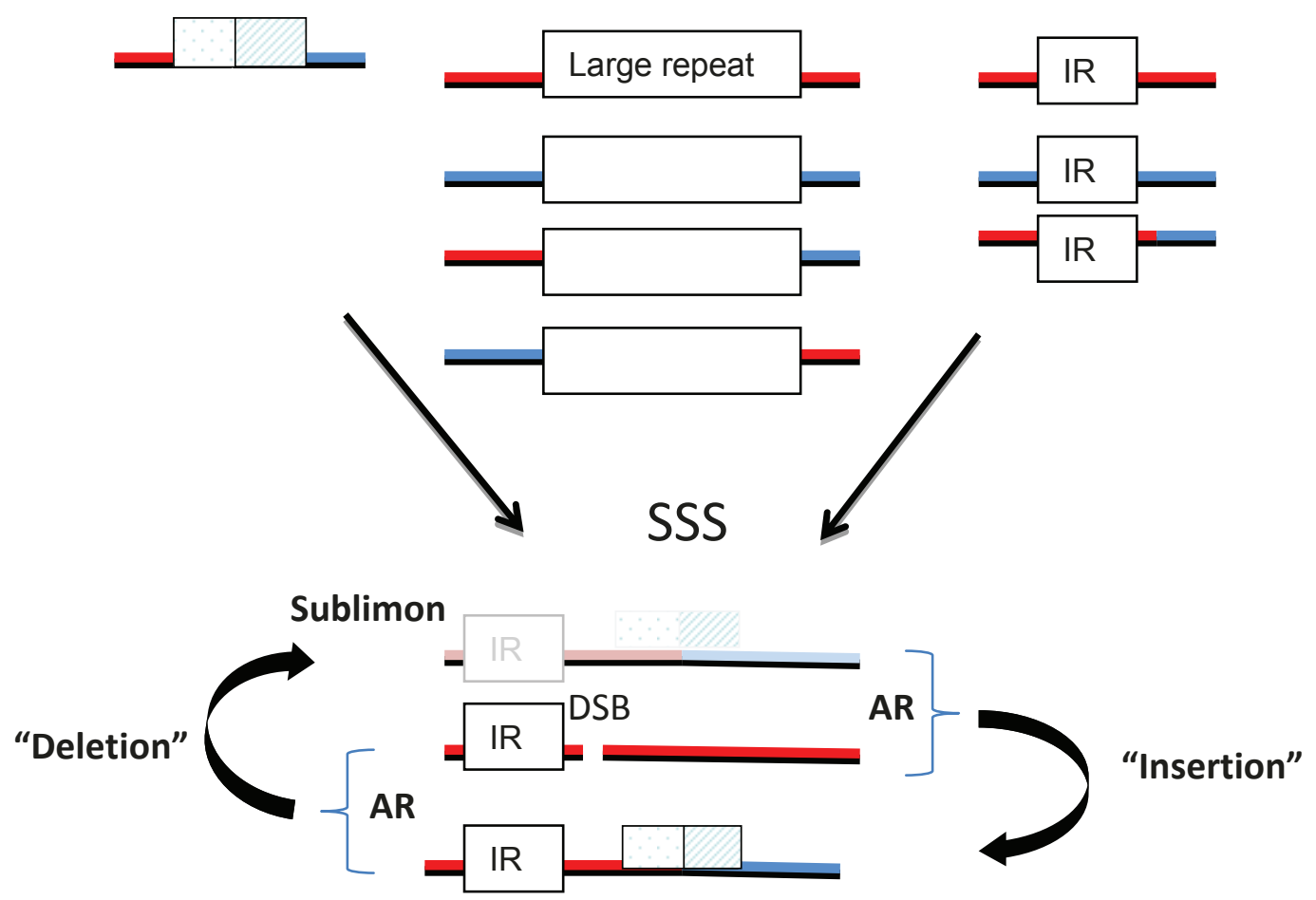

Figure 9 Modeling alternative double-strand break repair outcomes. Sequence analysis suggests three means of double-strand break (DSB) repair, resulting in evidence of nonhomologous end-joining (NHEJ), reciprocal recombination at large repeats and asymmetrical recombination at intermediate repeats (IRs). Asymmetrical recombination activity at IRs residing on molecules containing gene chimeras can result in substoichiometric shifting (SSS) for relative copy number suppression or amplification of the genomic environment surrounding the chimera.

and intermediate repeats are annotated. Generated raw sequence data are available from the sequence read archive [GenBank:SRA039856].

The Illumina Genome Analyzer (Illumina, Inc) was used with paired-end reads of $36 \mathrm{bp}$, a mean library length of 220 and a standard deviation of about 20 . This length spanned approximately half of the repeats in the mitochondrial genome. Coverage for first-generation $m s h 1$ was about $600 \times$ and for advanced-generation $m s h 1$ it was about 200x. Reads of the Illumina Solexa Sequencing Technology paired-end sequencing for first- and advanced-generation msh1 (Col-0) mutants were mapped to the mitochondrial genome of Col-0 using Velvet software [40] and SHOREmap software [41], and recombination points were identified with a customized script designed to cluster paired-end reads whose ends map to regions separated by more than $1,000 \mathrm{bp}$. Clusters with more than 10 reads in both the first- and advanced-generation $m s h 1$ lines were selected, and putative repeats were identified by BLAST analysis [43] of the selected region against the mitochondrial map. Coverage analysis of the regions around the intermediate repeats was done using custom-made scripts. On the basis of observed behavior in both the first- and advanced-generation msh1 lines and DNA gel blot data, we determined the putative direction of strand invasion around the repeats. For nucleotide sequence analysis of the intermediate repeats in the msh1 mutants, we used a custom-made script to derive the consensus sequence.

Illumina Solexa Sequencing Technology paired-end sequence data for 80 ecotypes was produced by the Weigel laboratory at the Max Planck Institute for Developmental 
Biology (http://www.1001genomes.org/). Aligned sequence data against the C24 mitochondrial genome with SHOREmap version 0.5 software [41] allowed us to select of 72 ecotypes with more than $15 \times$ coverage. SNPs against C24 were calculated using custom-made scripts for regions with more than $5 \times$ coverage and nucleotide occurrence in more than $80 \%$ of the reads mapped. For phylogenetic analysis, a concatenated set of 1,059 SNPs distributed across the genome in both protein-coding regions and intergenic regions was considered for each ecotype. Poorly aligned regions and regions with gaps in most ecotypes were excluded from the analysis, and a total of 676 nucleotides were used for each ecoytpe. A maximum likelihood model was preferred over a parsimony approach, considering its statistical consistency. Using PhyML version 3.0 software [44], we constructed the tree with an HKY85 substitution model and a $\gamma$ distribution with four different categories, and one hundred bootstrap replicates were performed. The resulting trees are unrooted. Graphical phylogenetic trees were generated by using TreeDyn software [45]. Geographical maps were generated by using a custom-made script and Google Static Maps API version 2 (http://code.google.com/apis/maps/documentation/staticmaps/). Deletions were identified by analyzing the coverage data, and recombination polymorphisms were identified by grouping paired-end reads whose ends map to regions separated by more than 1,000 bp with a custom-made script. Data for the 80 ecotypes are available at "1001 Genomes: A Catalog of Arabidopsis thaliana Genetic Variation: MPICao2010: 80 Arabidopsis thaliana accessions" (http://www.1001genomes.org/projects/MPICao2010/index.html) [46].

\section{Additional material}

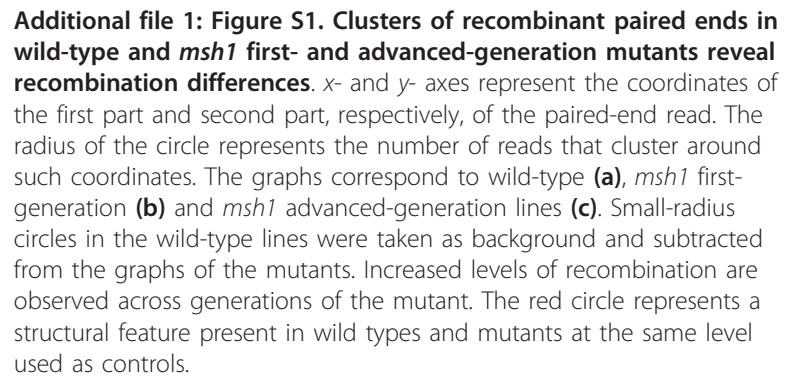

Additional file 2: Figure S2. Fate of genomic environments around repeats in $m s h 1$ mutants across generations. $y$-axis represents the percentage changes in coverage across each copy of the repeat with respect to wild type. The pink and red squares represent the recombinant forms gained in $m s h 1$ first- and advanced-generation lines. The turquoise and blue triangles represent the parental forms lost in msh 1 first and advanced generations. These were calculated as the difference in coverage of right versus left flanking regions of each repeat relative to wild type. A gradient, as well as a correspondence, between gain of recombinant and loss of parental forms is observed across generations.
Additional file 3: Table S1. Changes in coverage and putative strand invasion polarity for intermediate repeats in the $m s h 1$ mutant. Coverage data are shown for first- and advanced-generation mutants. The data show changes in coverage with respect to Col-0 for regions flanking each repeat, along with deduced order of strand invasion (strand polarity). Confirmation by gel blot analysis is indicated for several sites.

Additional file 4: Figure S3. Potential outcomes of double-strand break repair during break-induced replication. With the occurrence of a double-strand break (DSB) during replication, 5' to $3^{\prime}$ strand recession occurs, followed by strand invasion to a homologous duplex for reestablishment of the replication fork. (1) Occurrences of the DSBs at a unique region. (2) When the DSB occurs at a repeated region, two outcomes are possible: (a) strand invasion at homologous repeats or (b) strand invasion across different copies of the repeat to produce a new genomic environment as the recombinant MSH1 prevents DNA exchange beyond the region of sequence homology within the repeated sequence.

Additional file 5: Table S2. Primers used in the study.

\section{Abbreviations}

bp: base pair; kb: kilobase; Mbp: megabase pair; PCR: polymerase chain reaction; SNP: single-nucleotide polymorphism.

\section{Acknowledgements}

We acknowledge Hardik Kundariya for excellent technical assistance, and we thank Alan Christensen and Jeffrey Mower for valuable discussions throughout the study. This work was funded by grants from the National Science Foundation (MCB 0744104 and IOS 0820668) (to SAM).

\section{Author details}

${ }^{1}$ Center for Plant Science Innovation, University of Nebraska, N305 Beadle Center, Lincoln, NE 68588-0660, USA. ${ }^{2}$ Max Planck Institute for Developmental Biology, Spemannstr. 35-39, 72076 Tübingen, Germany. ${ }^{3}$ DuPont Agricultural Biotechnology, Experimental Station, Route 141 and Henry Clay Road, Wilmington, DE 19880-0357, USA.

\section{Authors' contributions}

JID participated in computational analysis, genome assembly, literature screening and manuscript preparation. MAM carried out PCR and gel blot hybridization analyses and genome mapping. YW, VS and YZX participated in mitochondrial genome sequencing experiments in msh1, Col-0, Ler, and C24. JC, JH and DW generated total genomic sequence from 72 Arabidopsis ecotypes. SAM designed and coordinated the study, participated in data analysis and prepared the manuscript. All authors read and approved the final manuscript.

\section{Conflicts of interests}

The authors declare that they have no competing interests.

Received: 5 September 2011 Accepted: 27 September 2011 Published: 27 September 2011

\section{References}

1. Bendich AJ: Structural analysis of mitochondrial DNA molecules from fungi and plants using moving pictures and pulsed-field gel electrophoresis. J Mol Biol 1996, 255:564-588.

2. Oldenburg DJ, Bendich AJ: Mitochondrial DNA from the liverwort Marchantia polymorpha: circularly permuted linear molecules, head-totail concatemers, and a 5' protein. J Mol Biol 2001, 310:549-562.

3. Sugiyama $Y$, Watase $Y$, Nagase $M$, Makita $N$, Yagura $S$, Hirai $A$, Sugiura M: The complete nucleotide sequence and multipartite organization of the tobacco mitochondrial genome: comparative analysis of mitochondrial genomes in higher plants. Mol Gen Genomics 2005, 272:603-615.

4. Backert $S$, Dörfel P, Lurz R, Börner T: Rolling-circle replication of mitochondrial DNA in the higher plant Chenopodium album (L.). Mol Cell Biol 1996, 16:6285-6294. 
5. Arrieta-Montiel MP, Mackenzie SA: Plant mitochondrial genomes and recombination. In Advances in Plant Biology: Plant Mitochondria. Edited by: Kempen F. New York: Springer; 2010:65-84

6. Allen JO, Fauron CM, Minx P, Roark L, Oddiraju S, Lin GN, Meyer L, Sun H, Kim K, Wang C, Du F, Xu D, Gibson M, Cifrese J, Clifton SW, Newton KJ: Comparisons among two fertile and three male-sterile mitochondrial genomes of maize. Genetics 2007, 177:1173-1192.

7. McNay JW, Chourey PS, Pring DR: Molecular analysis of genomic stability of mitochondrial DNA in tissue cultured cells of maize. Theor Appl Genet 1984, 67:433-437.

8. Shirzadegan M, Christey M, Earle ED, Palmer JD: Rearrangement, amplification, and assortment of mitochondrial DNA molecules in cultured cells of Brassica campestris. Theor App/ Genet 1989, 77:17-25.

9. Honda $\mathrm{H}$, Itoh $\mathrm{K}$, Hirai $\mathrm{A}$ : The heterogeneous composition of mitochondrial DNA in somatic hybrid calli and the relatively simple composition of such DNA in regenerated leaves. Jpn J Genet 1991, 66:279-289.

10. Schnable PS, Wise RP: The molecular basis of cytoplasmic male sterility and fertility restoration. Trends Plant Sci 1998, 3:175-180.

11. McCauley DE, Olson MS: Do recent findings in plant mitochondrial molecular and population genetics have implications for the study of gynodioecy and cytonuclear conflict? Evolution 2008, 62:1013-1025.

12. Backert S, Börner T: Phage T4-like intermediates of DNA replication and recombination in the mitochondria of the higher plant Chenopodium album (L.). Curr Genet 2000, 37:304-314.

13. Backert S: R-loop-dependent rolling-circle replication and a new model for DNA concatemer resolution by mitochondrial plasmid mp1. EMBO 2002, 21:3128-36.

14. Wolfe KH, Li WH, Sharp PM: Rates of nucleotide substitution vary greatly among plant mitochondrial, chloroplast, and nuclear DNAs. Proc Natl Acad Sci USA 1987, 84:9054-9058.

15. Palmer JD: Contrasting modes and tempos of genome evolution in land plant organelles. Trends Genet 1990, 6:115-120.

16. Arrieta-Montiel M, Lyznik A, Woloszynska M, Janska H, Tohme J, Mackenzie S: Tracing evolutionary and developmental implications of mitochondrial stoichiometric shifting in the common bean. Genetics 2001, 158:851-864.

17. Small ID, Isaac PG, Leaver CJ: Stoichiometric differences in DNA molecules containing the atpA gene suggest mechanisms for the generation of mitochondrial genome diversity in maize. EMBO J 1987, 6:865-869.

18. Janska H, Sarria R, Woloszynska M, Arrieta-Montiel M, Mackenzie SA: Stoichiometric shifts in the common bean mitochondrial genome leading to male sterility and spontaneous reversion to fertility. Plant Cell 1998, 10:1163-1180.

19. Johns C, Lu M, Lyznik A, Mackenzie S: A mitochondrial DNA sequence is associated with abnormal pollen development in cytoplasmic male sterile bean plants. Plant Cell 1992, 4:435-449.

20. Shedge V, Arrieta-Montiel M, Christensen AC, Mackenzie SA: Plant mitochondrial recombination surveillance requires unusual $\operatorname{Rec} A$ and MutS homologs. Plant Cell 2007, 19:1251-1264.

21. Arrieta-Montiel MP, Shedge V, Davila J, Christensen AC, Mackenzie SA: Diversity of the Arabidopsis mitochondrial genome occurs via nuclearcontrolled recombination activity. Genetics 2009, 183:1261-1268.

22. Abdelnoor RV, Yule R, Elo A, Christensen AC, Meyer-Gauen G, Mackenzie SA: Substoichiometric shifting in the plant mitochondrial genome is influenced by a gene homologous to MutS. Proc Natl Acad Sci USA 2003, 100:5968-5973.

23. Llorente B, Smith CE, Symington LS: Break-induced replication. Cell Cycle 2008, 7:859-864.

24. Maréchal A, Brisson N: Recombination and the maintenance of plant organelle genome stability. New Phytol 2010, 186:299-317.

25. Forner J, Weber B, Wiethölter C, Meyer RC, Binder S: Distant sequences determine $5^{\prime}$ end formation of cox3 transcripts in Arabidopsis thaliana ecotype C24. Nucleic Acids Res 2005, 33:4673-4682.

26. Satoh M, Kubo T, Mikami T: The Owen mitochondrial genome in sugar beet (Beta vulgaris L.): possible mechanisms of extensive rearrangements and the origin of the mitotype-unique regions. Theor Appl Genet 2006, 113:477-484.

27. Kubo T, Newton KJ: Angiosperm mitochondrial genomes and mutations. Mitochondrion 2008, 8:5-14
28. Beck JB, Schmuths $H$, Schaal BA: Native range genetic variation in Arabidopsis thaliana is strongly geographically structured and reflects Pleistocene glacial dynamics. Mol Ecol 2008, 17:902-915.

29. Alverson AJ, Zhu S, Rice DW, Sloan DB, Palmer JD: The mitochondrial genome of the legume Vigna radiata and the analysis of recombination across short mitochondrial repeats. PLOS One 2011, 6:e16404.

30. Hellberg ME: No variation and low synonymous substitution rates in coral mtDNA despite high nuclear variation. BMC Evol Biol 2006, 6:24.

31. Abdelnoor RV, Christensen AC, Mohammed S, Munoz-Castillo B, Moriyama H, Mackenzie SA: Mitochondrial genome dynamics in plants and animals: convergent gene fusions of a MutS homolog. J Molec Evol 2006, 63:165-173.

32. Sandhu AP, Abdelnoor RV, Mackenzie SA: Transgenic induction of mitochondrial rearrangements for cytoplasmic male sterility in crop plants. Proc Natl Acad Sci USA 2007, 104:1766-1770.

33. Escote LJ, Gabay-Laughnan SJ, Laughnan JR: Cytoplasmic reversion to fertility in cms-S maize need not involve loss of linear mitochondrial plasmids. Plasmid 1985, 14:264-267.

34. Bellaoui M, Martin-Canadell A, Pelletier G, Budar F: Low-copy-number molecules are produced by recombination, actively maintained and can be amplified in the mitochondrial genome of Brassicaceae: relationship to reversion of the male sterile phenotype in some cybrids. Mol Gen Genet 1998, 257:177-185.

35. Woloszynska M, Trojanowski D: Counting mtDNA molecules in Phaseolus vulgaris: sublimons are constantly produced by recombination via short repeats and undergo rigorous selection during substoichiometric shifting. Plant Mol Biol 2009, 70:511-522.

36. Shedge V, Davila J, Arrieta-Montiel MP, Mohammed S, Mackenzie SA: Extensive rearrangement of the Arabidopsis mitochondrial genome elicits cellular conditions for thermotolerance. Plant Physiol 2010, 152:1960-1970.

37. Li J, Chory J: Preparation of Arabidopsis DNA. In Arabidopsis Protocols. Edited by: Martinez-Zapater J, Salinas J. Totowa, NJ: Humana Press; 1998:55-60.

38. Janska $H$, Mackenzie SA: Unusual mitochondrial genome organization in cytoplasmic male sterile common bean and the nature of cytoplasmic reversion to fertility. Genetics 1993, 135:869-879.

39. Pop M, Phillippy A, Delcher AL, Salzberg SL: Comparative genome assembly. Brief Bioinformatics 2004, 5:237-248.

40. Langmead B, Trapnell C, Pop M, Salzberg SL: Ultrafast and memoryefficient alignment of short DNA sequences to the human genome. Genome Biol 2009, 10:R25.

41. Ossowski S, Schneeberger K, Clark RM, Lanz C, Warthmann N, Weigel D: Sequencing of natural strains of Arabidopsis thaliana with short reads. Genome Res 2008, 18:2024-2033.

42. Lohse M, Drechsel O, Bock R: OrganellarGenomeDRAW (OGDRAW): a tool for the easy generation of high-quality custom graphical maps of plastid and mitochondrial genomes. Curr Genet 2007, 52:267-274.

43. Altschul SF, Gish W, Miller W, Myers EW, Lipman DJ: Basic local alignment search tool. J Mol Biol 1990, 215:403-410.

44. Guindon S, Gascuel O: A simple, fast, and accurate algorithm to estimate large phylogenies by maximum likelihood. Systematic Biol 2003, 52:696-704.

45. Chevenet F, Brun C, Bañuls AL, Jacq B, Christen R: TreeDyn: towards dynamic graphics and annotations for analyses of trees. BMC Bioinformatics 2006, 7:439.

46. Cao J, Schneeberger K, Ossowski S, Günther T, Bender S, Fitz J, Koenig D, Lanz C, Stegle O, Lippert C, Wang X, Ott F, Müller J, Alonso-Blanco C, Borgwardt K, Schmid KJ, Weigel D: Whole-genome sequencing of multiple Arabidopsis thaliana populations. Nat Genet .

doi:10.1186/1741-7007-9-64

Cite this article as: Davila et al:: Double-strand break repair processes drive evolution of the mitochondrial genome in Arabidopsis. BMC Biology 2011 9:64. 